\title{
A System Behavior Analysis Technique with Visualization of a Customer's Domain
}

\author{
Shoichi Morimoto \\ School of Industrial Technology, Advanced Institute of Industrial Technology \\ 1-10-40, Higashi-oi, Shinagawa-ku, Tokyo, 140-0011, Japan \\ morimoto-syoichi@aiit.ac.jp
}

\begin{abstract}
Object-oriented analysis with UML is an effective process for software development. However, the process closely depends on workmanship and experience of software engineers. In order to mitigate this problem, a precedence effort, scenario-based visual analysis, has been proposed. The technique visualizes a customer's domain, thus it enables requirement analyzers and customers to communicate smoothly. The customers themselves can schematize their workflows with the technique. Consequently, the analyzers and customers can easily and exactly derive use case models via the collaborative works. Thus, this paper proposes a technique to advance the analysis further, inheriting such advantages. The extended technique can analyze initial system behavior specifications. The customers can also join and understand system behavior analysis, thus they can easily decide on specifications for developing systems.
\end{abstract}

Keywords: Activity diagrams, Model-based development.

\section{Introduction}

Requirement analysis and specifications for software are important factors to make success of software development and because quality of the analysis affects quality of the software, it is the most important process. Thus, various analysis techniques were proposed; especially, Object-Oriented Analysis (OOA) with $\mathrm{UML}^{1} 1$ is most widely used to model a domain of customers. After the modeling, customers and developers can understand and analyze the domain and systematically decide requirement specifications [6]. However, because the developers must fully analyze a domain of customers based on OOA, quality of the analysis is dependent on their capability.

In order to mitigate the problem, Scenario-based Visual Analysis, SVA for short, was proposed [3, 2]. In SVA, analyzers and customers can analyze requirements in a domain and elicit use cases from very simple workflow scenarios cooperatively. That is to say they can easily understand, schematize and analyze the domain in a much simpler manner than using UML. On the other

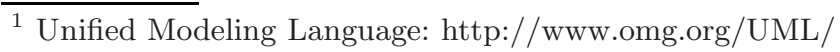


hand, difficulty and quality of system behavior analysis in OOA with UML is dependent on workmanship and experience of designers likewise. Therefore, this paper proposes a system behavior analysis technique utilizing resources which are generated in the SVA processes. One can model not only use cases but also system behavior with the technique. Moreover, the technique visualizes a domain of customers, thus it enables designers and customers to communicate smoothly. Consequently, the customers can easily and exactly decide requirements to the developers.

\section{Process of the System Behavior Analysis Technique}

We herein explain the process of SVA and the system behavior analysis.

\subsection{The Process of the Scenario-Based Visual Analysis}

SVA can be adaptable to the requirements phase of object-oriented software development. That is, use case diagrams can be obtained via the collaborative works on the process. Use cases and actors are generally found out from a conceptual map, named business bird's eye view (BEV), through arranging icons which indicate subjects, verbs, and nouns in workflow scenarios. A software tool named SVA editor is also provided to support the operations [3]. Analyzers can systematically perform the analysis and customers can easily join to the process. Furthermore, in the last phase of the process, both of them can collaboratively and visually decide which part of the tasks in the scenario should be implemented as software on a BEV.

In SVA, analyzers use workflow scenarios in order to capture a customer's business domain. A BEV is created from the workflow scenarios to obtain a conceptual map of the domain. The BEV will be arranged to clarify the whole structure of the elements which constitutes the workflow. Finally, use case diagrams will be elicited from the BEV. The process of SVA is performed as following steps;

1. Customers describe workflow scenarios.

2. Analyzers form an initial BEV from the scenarios.

3. The analyzers arrange the BEV to cluster verbs.

4. The analyzers and the customers analyze roles of the subjects.

5. The analyzers consider the system boundary.

6. The analyzers obtain use case diagrams.

SVA defines some rules to write workflow scenarios as follows.

- Use simple sentences with one verb and several nouns which might involve articles, propositions, and adjectives. Other parses, such as adverbs, can be used but are not analyzed in SVA.

- Use active sentence. Do not use passive voice. The order of the words is such like Subject-Verb-Objects. 
Since task statements of customers are described in the workflow scenarios using simple natural language, they can easily confirm the correctness of the contents.

The analyzers form a BEV from each sentence in the workflow scenarios. Rectangle icons and oval icons are used to stand for nouns and verbs, respectively. A subject and the other icons are connected by lines from a verb. The line to the subject is drawn as a solid line and lines to the other icons are drawn as broken lines.

After all the sentences in the workflow scenarios have been visualized on the $\mathrm{BEV}$, the analyzers synthesize them. Same nouns are shared by every sentence in which they are used. Verbs are not shared even if the same verbs appear in several sentences.

After the synthesis, the analyzers rearrange elements so that the BEV will become legible. This arrangement also produces a semantic relation structure on the conceptual map. During this arrangement to clarify, the analyzers have to take care to find out semantic clusters of verb icons. This process is necessary to analyze roles of subjects in the next step where noun icons will be analyzed.

In the next step, the analyzers abstract subjective noun icons as roles of tasks. If a subjective noun icon is connected from some clusters of verbs, the subjective noun has some different roles. In such cases, a single subject is decomposed to multiple roles. Each of them is reconnected to the verb clusters.

After the role analysis, both the analyzers and customers decide a system boundary on the rearranged BEV. This decision is made by both the analyzers and the customers cooperatively. The boxed line is used to indicate what part of the tasks is developed to be a software system. After the boundary is drawn, the analyzers have to consider what nouns should be moved into the boundary. For example, they must decide whether or not physical objects are implemented in software. In such situations, the editable map, i.e., the BEV acts as a communication tool between the analyzer and customer. They can have a visual support, i.e., the map, to discuss about the scope of the system which is going to be developed.

Generating a use case diagram is the final step of SVA. The way how to elicit actors and use cases is very simple. The role icons connecting to the verbs on the system boundary line and located outside of the box become actors. The verbs on the boundary which connect to the actors become use cases.

You can get the further details of the SVA process from the references [3,2].

\subsection{Artifacts of the System Behavior Analysis Technique}

In the system behavior analysis of OOA with UML, interaction or activity diagrams are generally used. Interaction diagrams illustrate how objects interact via messages. Activity diagrams are typically used for business process modeling, for modeling the logic captured by a single use case or usage scenario, or for modeling the detailed logic of a business rule [1. Because activity diagrams are closely related with scenarios in natural language and are suitable for system behavior analysis, the objective of our technique is to design activity diagrams from the results in SVA (i.e., workflow scenarios, a BEV, and use case diagrams). 


\subsection{The Procedure of the System Behavior Analysis Technique}

The system behavior analysis technique effectively utilizes the artifacts of SVA. Designers first select a target use case from an elicited use case diagram in SVA. Secondly, the designers extract the source verb icon of the selected use case and all noun icons which are connected with the verb icon from the BEV. In the third step the designers and customers cooperatively analyze activities from the extracted icons and the source sentence of the icons in the workflow scenario. Next the designers draw partitions of an activity diagram based on the actors which are connected with the selected use case in the use case diagram. Then the designers set the elicited activities on the partition which is drawn from the actor of the activity's subject in chronological order. The designers repeat the above steps to all use cases in the use case diagram. The following is the procedure of the analysis.

1. After having used SVA, select a use case in a use case diagram.

2. Extract the source verb icon of the selected use case and all noun icons which are linked with the verb icon in the BEV.

3. Elicit activities from the extracted icons and the source sentence of the icons in the workflow scenario.

4. Draw partitions from actors which are linked with the selected use case in the use case diagram.

5. Put the activities on the corresponding partition in chronological order.

\section{Application}

In order to intelligibly demonstrate the steps of the process in detail, we present an actual consulting example. The business domain of this example is a hospital, where a new software system is needed to support their daily tasks. Especially, the staff's demand is that they want to develop the online medical record chart system for doctors.

\subsection{Use Case Analysis}

The objective of this paper is not to show the use case analysis of SVA, thus we show only the outline of the SVA phase.

The workflow scenario on the next page Fig. 1 shows the business domain of the first medical examination. The parts which are surrounded by square brackets denote modifications in the revision phase of SVA. These parts were elicited by collaborative discussion of the analyzers and customers. First, all the workflows were modeled into BEVs. Secondly, the BEVs were synthesized into one diagram. Thirdly, the verb icons were grouped and roles of the subjective nouns were analyzed in the synthesized BEV. The BEV on the next page Fig. 2 was finally modeled from the workflow scenario. The verb icons were classified into the clusters (A), (B), (C), (D), (E), (F), (G), (H), (I), (J), (K), and (L). 
1. The patient submits the insurance card to the receptionist.

2. The receptionist inquires of the patient the symptom.

3. The receptionist decides the department from the symptom.

4. The receptionist makes the patient fill the application.

5. The receptionist makes the medical chart from the application [and the insurance card].

6. [The receptionist makes the consultation card from the insurance card].

7. The receptionist brings the medical chart to the surgery of the department.

8. The receptionist hands the nurse in the surgery the medical chart.

9. The nurse calls the patient in the waiting room.

10. The patient enters the surgery.

11. The doctor examines the patient.

12. The doctor gives the medical treatment to the patient.

13. The doctor writes the medical treatment to the medical chart.

14. The patient leaves the surgery.

15. The patient goes the waiting room.

16. The nurse brings the medical chart to the reception.

17. The receptionist calculates the medical bill [from the medical treatment in the medical chart].

18. The receptionist calls the patient in the waiting room.

19. The receptionist charges the patient the medical bill.

20. The patient pays the receptionist the medical bill.

21. The receptionist hands the patient the consultation ticket [and the insurance card].

22. The receptionist puts the medical chart on the cabinet.

Fig. 1. The workflow scenario of the first medical examination

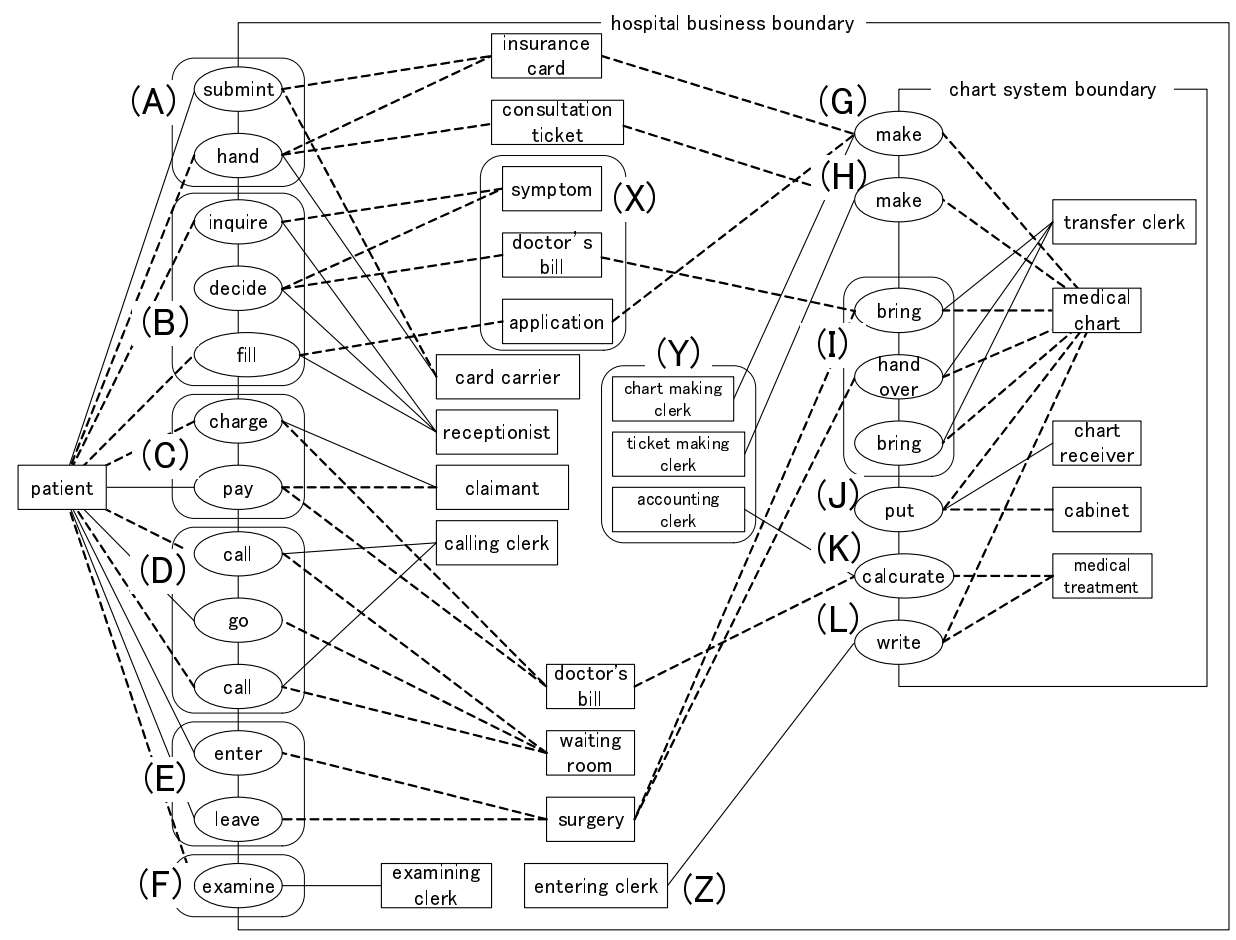

Fig. 2. The BEV of the hospital business 
The subjective nouns which are linked with two or more verbs were decomposed into some roles. Consequently, the use case diagram in Fig. 3 was also composed from the chart system boundary on Fig. 2. Use cases are the verb clusters $(\mathrm{G})$, $(\mathrm{H}),(\mathrm{K})$, and $(\mathrm{L})$ on the boundary. Actors are the roles $(\mathrm{Y})$ and $(\mathrm{Z})$ which are connected to the verb clusters with the solid line. Two actors and four use cases are elicited and named adequately. The foregoing operations are the SVA outline.

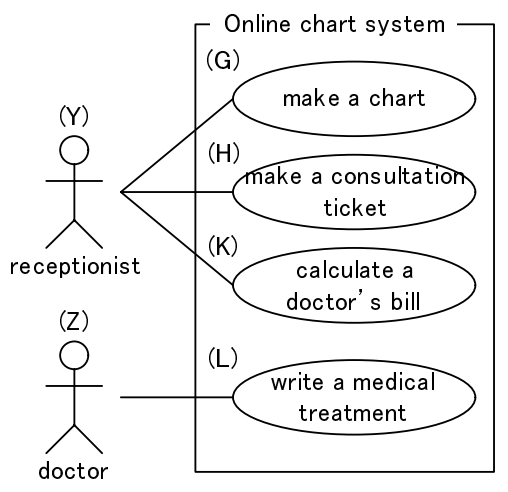

Fig. 3. The use case diagram of the online medical record chart system

\subsection{System Behavior Analysis}

We analyze the system behavior from the workflow, the BEV, and the use case diagram of Section 3.1.

First, we select the use case "make a chart." This use case was obtained from the part $(\mathrm{G})$ in the BEV. The verb icon is "make" on the part $(\mathrm{G})$ of Fig. 2 and the noun icons which are directly linked to it are "insurance card," "application," and "medical chart." These are the first and second processes of the system behavior analysis mentioned in Section 2.3.

Next, see the source sentence of the icons in the scenario Fig. 1. The sentence corresponds to " 5 . The receptionist makes the medical chart from the application and the insurance card." That is, it is clarified that the information of "application" and "insurance card" is required in order to make a medical chart. Therefore, the activities "input the information on an application" and "input the information on an insurance card" are identified. We used the nouns "application" and "insurance card," however the noun "medical chart" is not yet used. The business domain of this example is the first medical examination, thus the patient visits the hospital surely for the first time. Because there is no chart of the patient, the receptionist must make a new chart for the patient. That is, before the foregoing activities, the activity "make a new chart data" is required. The above operation is the third process of the system behavior analysis.

Finally, make a partition from the actor linked to the use case, i.e., "receptionist" and put the activities on the partition in chronological order. In consequence, the activity diagram in Fig. 目is composed. 


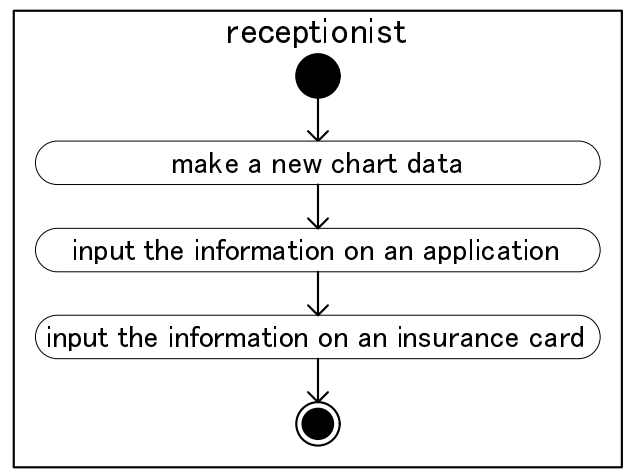

Fig. 4. The activity diagram of the use case "make a chart"
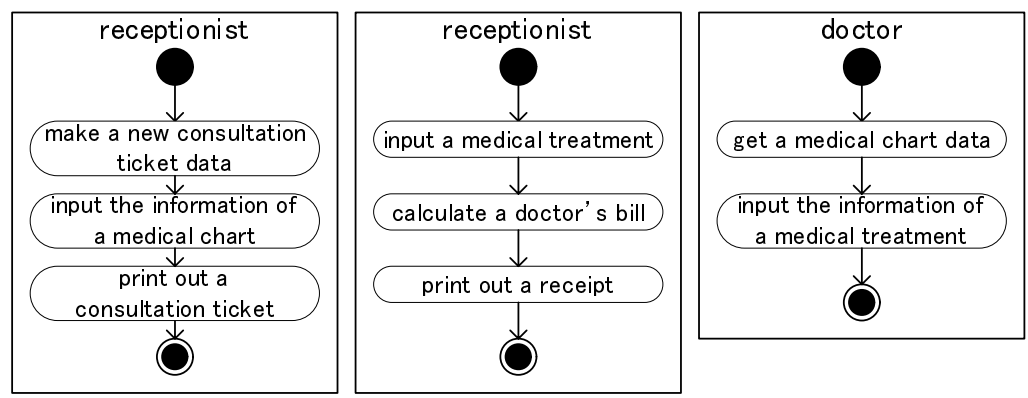

Fig. 5. The activity diagrams of the other use cases

Similarly, we analyze the use case "make a consultation ticket." This use case was obtained from the part $(\mathrm{H})$ in the BEV. The verb icon is "make" on the part $(\mathrm{H})$ of Fig. 2 and the noun icons which are directly linked to it are "consultation ticket" and "medical chart." The source sentence corresponds to "6. The receptionist makes the consultation card from the insurance card." The information of "medical chart" is required in order to make a consultation ticket. Therefore, the activity "input the information on a medical chart" is clarified. Because there is no consultation ticket of the patient like the above analysis, the activity "make a new consultation ticket data" is required. Moreover, if you pursue the connection of the icon "consultation ticket," it will be clarified that the receptionist must hand the patient the actual object, i.e., the ticket. Accordingly, the receptionist must print out the ticket with the online chart system; the activity "print out a consultation ticket" is identified. Since the third process of the system behavior analysis is a group work, the designers can easily get customer's consent about such activities. The other use cases can be analyzed in the same procedure likewise. The diagrams are shown in Fig. 5.

Moreover, the technique is adaptable to the business modeling [5]. SVA can also analyze business use cases. If the boundary for the hospital business is drawn 
in Fig. 2, the business use case diagram is formed in the same way. Similarly, a business activity diagram can be elicited from the workflow scenario. The subject in each workflow may become a partition of a business activity diagram and each workflow sentence excepted the subject clause may become a business activity.

\section{Concluding Remarks}

In this paper, we have proposed a system behavior analysis technique utilizing the scenario-based visual analysis. Software designers can obtain activity diagrams according to the process of the technique. Since the technique utilizes the scenario-based visual analysis, the designers can understand customer's business domain. Moreover, since the rules of the scenario description are very simple, the customers who fully understand the business domain can write the workflow scenarios. Consequently, they can have common understanding via the group works. That is, they can easily decide specifications on software development.

Henceforth, we will apply the technique to further practical subjects. The activity diagrams of the example were not complex; they do not use condition or decision. Moreover, the technique designs only activity diagrams now. If class diagrams can be elicited from workflow scenarios, sequence and state machine diagrams can also be designed. In the business bird's eye view, designers can easily decide classes, because noun factors in the customer's domain are clarified. However, in order to identify attributes and methods of classes, it may be necessary to add further information, e.g., a picture. In the case of the example in this paper, maybe the picture of the medical chart is required for class design. We are improving the technique and developing a tool to aid this idea.

Acknowledgement. My special thanks are due to Dr. Chubachi for his advice.

\section{References}

1. Ambler, S. and Jeffries, R.: Agile Modeling, Wiley (2002)

2. Chubachi, Y., Kobayashi, T., Matsuzawa, Y., and Ohiwa, H.: Scenario-Based Visual Analysis for Use Case Modeling, IEICE Transactions on Information and Systems, Vol. J88-D1, No.4 (2005) 813-828 (in Japanese)

3. Chubachi, Y., Matsuzawa, Y., and Ohiwa, H.: Scenario-Based Visual Analysis for Static and Dynamic Models in OOA, Proceedings of the IASTED International Conference on Applied Modelling and Simulation (AMS 2002), ACTA Press (2002) 495-499

4. Jacobson, I., Booch, G., and Rumbaugh, J.: The Unified Software Development Process, Addison-Wesley (1999)

5. Jacobson, I., Ericsson, M., and Jacobson, A.: The Object Advantage - Business Process Reengineering with Object Technology, Addison-Wesley (1996)

6. Kruchten, P.: The Rational Unified Process: An Introduction, Addison-Wesley (2003) 